number of fledglings produced per nest was about half. So increased winter food affected date of laying but not, per se, the number of fledglings produced per nest.

How does extra food increase the survival rates of nestling crows? Predation of the eggs and desertion by the adults were the main factors responsible. Krebs has pointed out that artifically increased winter food has no effect on the number of territorial breeding pairs of great tits during the following spring (Ecology, 52, 2; 1971). Those nests that were closer together than $45 \mathrm{~m}$ suffered $12 \%$ more predation (mostly by weasels) than did those nests spaced over $45 \mathrm{~m}$ apart. Carrion crows are their own chief predatorsthey prey to a significant extent upon the eggs and nestlings of their own kind. With cannibalism as the regulatory mechanism, the ultimate limiting factor for the number of nestlings reared is the dispersion of food within the territory. An abundance of food close to the nest means that the clutch is left unattended for shorter intervals than if the food is widely scattered. To remain near the nest breeding crows limit their territory size. The lower limit to territory size depends upon the amount of food available and the upper limit depends upon its dispersion and the ability of the pair to defend its nest.

\section{Cyclic AMP and pattern formation}

\section{from Paul Epstein}

By staining with fluorescent antibody specific for cyclic AMP, Pan et al. (Proc. natn. Acad. Sci. U.S.A., 71, 1623; 1974) have been able to observe the distribution of cyclic AMP in both the unicellular amoebae and the multicellular pseudoplasmodium of Dictyostelium discoideum and several related species of cellular slime mould. Cyclic AMP was first shown to be important in the life cycle of $D$. discoideum when it was isolated as the naturally occurring acrasin, or chemotactic agent by which the vegetative amoebae signal each other to aggregate (Barkley, Science, 165, 1133; 1969). Now the studies by Pan et al. suggest that cyclic AMP is important not only for aggregation, but for determination, or paittern formation, as well.

In the cellular slime moulds, once the aggregation process is completed, the amoebae form a pseudoplasmodium, or grex, which, depending on environmental conditions, may migrate for an indefinite period before beginning to differentiate into two morphologically and functionally distinct cell types-spore cells and stalk cells. By staining with vital dyes, Bonner (The Cellular
Slime Molds, second ed., Princeton University Press, Princeton, New Jersey, 1967) showed that the celits in the anterior third of the grex become stalk cells, whereas the cells in the remaining posterior portion become spore cells.

The cells in the grex are not predetermined, however, since a grex which is sliced transversely can form two normal fruiting bodies, containing the normal proportions of spore and stalk cells (Raper, J. Elisha Mitchell scient. Soc., 56, 241; 1940). This observation indicates that the cells can recognise their position within the grex, and somehow alter their developmental fate accordingly.

How do the cells know their position in a field? One theory is that they respond to gradient(s) of concentration of small molecule(s) (Wolpert, $J$. theoret. Biol., 25, 1; 1969). More recently, McMahon developed a theory of pattern formation using $D$. discoideum as a model, which holds that a shanp concentration boundary of a small molecule can develop through its regulation by contact-sensing molecules on the plasma membranes of cells in the field (McMahon, Proc. natn. Acad. Sci. U.S.A., 70, 2396; 1973). These regulatory molecules would be the macromolecules which synthesise and degrade the small molecule. In this case, the polarity of the morphogenetic field is determined by the boundary of the small molecule, which in turn is determined by the distribution of contact-sensing molecules.

Although one theory invokes a gradient and the other a boundary, both models require non-uniform distribution of a small molecule to determine the pattern of development. The results of Pan et al. suggest that cyclic AMP might be one such small molecule which determines pattern formation in $D$. discoideum. Pan et al. showed that as the pseudoplasmodium was allowed
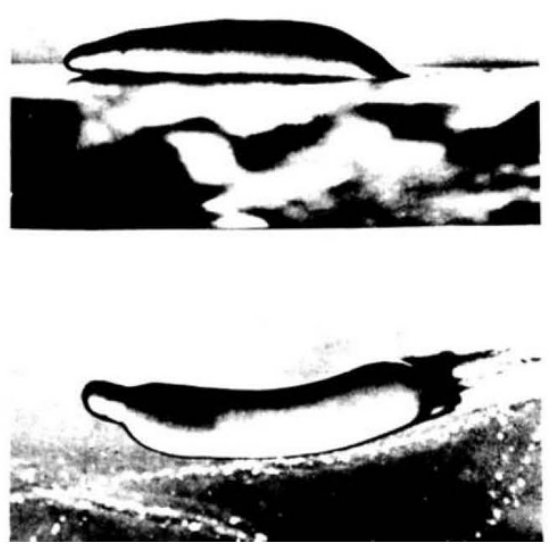

Migrating grex of Dictyostelium discoideum seen from the side and from above. From Developmental Biology by Nelson Spratt. to migrate, a distribution of cyclic AMP arose such that the higher concentration was found in the anterior, pre-stalk region. In some cases, they observed an abrupt boundary of cyclic AMP concentration between the anterior and posterior portions of the grex, as theorised by McMahon.

It is interesting to consider two earlier observations which bear on pattern formaltion and differentiation in $D$. discoideum, and to see how they fit with the idea that pattern formation in the grex is determined by the distribution of cyclic AMP. Bonner (Proc. natn. Acad. Sci. U.S.A., 65, 110; 1970) subjected isolated amoebae to high concentrations of cyclic AMP and found that they developed into stalk cells. From this observation, he concluded that cyclic AMP functions as an inducer of stalk cell differentiation. The observation of Pan et al. is consistent with this conclusion.

The other observation was made by Raper (J. Elisha Mitchell scient. Soc., 241, 1940), who sliced a pseudoplasmodium transversely into four sections. Section one, the anterior tip, could be made to migrate for various times. If induced to begin fruiting body formation immediately, without any resumption of migration, this section developed into an extremely abnormal fruiting body, bearing very few or no spores, and an excessively heavy stalk. If 3-6 $\mathrm{h}$ of migration were allowed before fruiting formation began, the resulting structure was less abnormal but still bore fewer spores and more stalk. If 24 $\mathrm{h}$ of migration were allowed, the tip gave rise to a completely normal fruiting body, with the correct proportion of spore and stalk cells. Hence, Raper demonstrated that the cells at the anterior tip of a pseudoplasmodium are determined to become stalk cells, and that this determined state is altered progressively as the tip migrates.

In accord with this observation, Pan et al. found that the distribution of cyclic AMP in the grex becomes more apparent as it migrates. Likewise, McMahon, in his theoretical treatment. showed the boundary of cyclic AMP concentration arising near one end of the field, and moving along the field with time. McMahon calculated that by about $3-5 \mathrm{~h}$ the boundary becomes stabilised at a position one-third of the way down the field; that is, at the position which would give rise to the normal proportion of spore and stalk cells.

Although substantial proof is required before one can state that cyclic AMP is responsible for pattern formation in $D$. discoideum, the results of Pan et al. at least suggest such a function. If further studies provide proof, this would represent the first identification of a small molecule responsible for pattern formation. 